Degenerate Fermi gases Tunneling, periodic potentials Josephson effects

\title{
Anisotropic Ginzburg-Landau and Lawrence-Doniach Models for Layered Ultracold Fermi Gases
}

\author{
Mauro Iazzi, ${ }^{1}$ Stefano Fantoni, ${ }^{2}$ and Andrea Trombettoni ${ }^{3,1}$ \\ ${ }^{1}$ SISSA, Via Bonomea 265, I-34136 Trieste, Italy \\ ${ }^{2}$ ANVUR, P.za Kennedy 20, I-00144 Roma, Italy \\ ${ }^{3}$ CNR-IOM DEMOCRITOS Simulation Center, \\ Via Bonomea 265, I-34136 Trieste, Italy
}

\begin{abstract}
We derive and study the anisotropic Ginzburg-Landau and Lawrence-Doniach models describing a layered superfluid ultracold Fermi gas in optical lattices. We compute from the microscopic model the Josephson couplings entering the LawrenceDoniach model across the crossover BCS-BEC passing from the 3D isotropic case to the quasi-2D one, showing that a model with only nearest-neghbour Josephson coupling is not adequate at the unitary limit (since the pairs have a diameter larger than the interlayer distance). We also show that the effective anisotropy of the system is strongly reduced ad the unitary limit. Finally we obtain a relation between the interlayer Josephson couplings and the Ginzburg-Landau masses: we find that using only couplings between adjacent planes is correct in the BEC side, while at the unitary limit one has to use also next-nearest neighboring couplings.
\end{abstract}

PACS numbers: $74.50 .+\mathrm{r}$ 


\section{INTRODUCTION}

The presence and nature of the superfluid and superconducting transition in systems of different dimensionality is a widely studied and interesting topic. It is well known that the transition in two and three dimensions is radically different, as the first is governed by a Kosterlitz-Thouless mechanism of topological origin (the unbinding of vortices) and the second is the paradigmatic example of the symmetry breaking well described, at least qualitatively, by mean-field theories. This is one of the main reasons of the wide interest in the study of materials with properties in-between the two, i.e. layered superfluids and superconductors. Their phenomenological properties are usually well described by anisotropic effective models: a successful example is given by the Lawrence-Doniach (LD) model [1] (in which the discrete layers are weakly coupled through Josephson terms) describing the electrodynamics of layered superconductors and copper oxides [2]. Another related way to introduce the effect of the layering is to consider a Ginzburg-Landau (GL) theory having anisotropic masses (eventually phenomenologically determined) [2]. A well known feature of these phenomenological models is the fact that they work well even if the underlying microscopic mechanisms are not known (as it occurs for high- $T_{c}$ cuprates).

In this paper we study and compare the anisotropic GL and LD models describing a layered superfluid ultracold Fermi gas in optical lattices: the coefficients of these phenomenological models are derived from the microscopic underlying Hamiltonian, the parameters of which, e.g. the strength of the optical lattices, can be tuned with high accuracy in realistic experimental setups. We determine the mass tensor in the GL theory as a function of lattice anisotropy, filling and interaction, showing that near the unitary limit the effective anisotropy of the masses is significantly reduced. By tuning the strength of the optical lattices, the dimensionless anisotropy parameter $\gamma$ in realistic setups can vary in a wide range of values: $\gamma \sim 1-200$ (for comparison with layered superconductors, we remind that $\gamma \sim 7$ for YBCO and $\gamma \sim 150$ for BSCCO [2]). We also derive the LD model, discussing a relation between the interlayer Josephson energy couplings in the LD model and the masses in the GL model. shown to be equivalent to the anisotropic GL theory in the BEC limit, while in the BCS side next-nearest-neighbor couplings have to be taken into account, and a 


\section{LAYERED SUPERCONDUCTORS AND SUPERFLUIDS}

The first layered superconductor was realized alternating layers of graphite and alkali metals $[5]$, with very low critical temperatures $(\approx 0.1 \mathrm{~K})$. It was followed by the discovery of naturally occurring compounds of transition-metal dichalcogenides layers (such as $\mathrm{TaS}_{2}$ ) intercalated with organic, insulating molecules [3] and the creation of artificial samples with alternating layers of different metals, having a different transition temperature [4], both systems with a higher critical temperature and thus more practical to study. The nature of the transition was found to be three dimensional [6], and the systems were shown to be very well described by an anisotropic GL free energy at and above the transition, where the coherence length diverges. At lower temperatures where the length $\xi$ becomes comparable with the plane separation, the presence of a layer structure becomes important. To describe this Lawrence and Doniach proposed a tight-binding approximation [1] able to describe the crossover between the $2 \mathrm{D}$ behaviors of essentially decoupled planes to the $3 \mathrm{D}$. being the interlayer distance and $\xi_{\perp}$ the coherence length perpendicular to the planes). On this line the critical magnetic field parallel to the planes diverges in the LD model. To remove this divergence one has to include the effects of Pauli paramagnetism and spin-orbit coupling [6].

Another very important class of superconductors having a layered structure is the one of cuprates, discovered in 1986 by Bednorz and Müller [7]. copper oxide planes alternated to an insulating lattice with a perovskite-like structure. reach temperatures as high as $130 K$. Their layered structure makes the behavior of cuprates strongly anisotropic and the dimensional crossover temperature is in general very near the critical temperature for the resistive transition [2]: despite the lack of a definitive consensus on the mechanism responsible for the superconductivity in the cuprates, it is by now established that the LD model successfully describe the transition and the electromagnetic response of cuprates [2, 8].

The study of the dimensional crossover in cuprates is not in general an easy task: on one hand, the smallest barrier attainable is that of a single plane of an insulating material, on the other hand inserting several planes is a delicate process as the separation can become nonuniform [8]. From this point of view, ultracold atoms (and in particular ultracold fermions) in optical lattices provide a favorable setting: the tunneling rate $t$ can be controlled by adjusting the optical lattice parameters $[9,10]$ and the on-site energy coefficient $U$ can be controlled through Feshbach resonances [10]. The ratio $U / t$ can be then controlled with 
high precision, as well as the geometry and the anisotropy of the system. respect to all other energy scales), the gas in the center is well described by an essentially homogeneous model. For ultracold bosons, optical lattices have been used to induce a Mott-insulator/superfluid quantum phase transition [11] and to study Josephson dynamics in many- and two- wells settings [12, 13]. The study of ultracold fermions in optical lattices also stimulated a huge amount of experimental work [14-19]. The low energy properties of ultracold fermions in optical lattices are described by Hubbard-like models [20, 21]: in presence of attractive interaction, with deep optical lattices one then can have a physical realization of the attractive Hubbard model [22 26].

Superimposing a 1D optical lattice to an ultracold Fermi gas gives raise to a stack of two-dimensional layers. Several equilibrium and dynamical properties of a Fermi superfluid in presence of a superimposed optical lattice have been theoretically studied [27-30]. Very recently, the evolution of fermion pairing from 3D to 2D was experimentally studied, showing the opening of a gap in the radiofrequency spectrum when the dimensionality is reduced [19]. The confinement of Fermi mixtures in 2D was also experimentally investigated [31 36], showing properties different from the 3D case [37]. The observation of a pairing pseudogap in a 2D Fermi gas, using momentum-resolved photoemission spectroscopy, has been reported [36].

An important advantage of the use of optical lattices is that when it is applied (say along the $z$ direction) then the interlayer coupling $t_{\|}$between 2D pancakes along $z$ can be easily modified by varying the strength of the optical lattice. Furthermore, if two additional optical lattices are added in the $x y$ plane, then the tunneling $t_{\perp}$ can be tuned separately from $t_{\|}$ and the layering can be tuned by varying the ratio $t_{\|} / t_{\perp}$.

\section{EFFECTIVE MODELS}

Near the critical temperature, the superfluid wavefunction $\Psi$ is small, so that the free energy difference $\delta F$ between the normal and superfluid state can be expanded in powers of $|\Psi|^{2}[38]$ : for a layered superconductor/superfluid $\delta F$ reads then

$$
\begin{gathered}
\delta F[\Psi]=\int d \vec{r}\left\{\frac{\hbar^{2}}{2 m_{\|}}\left(\left|\partial_{x} \Psi\right|^{2}+\left|\partial_{y} \Psi\right|^{2}\right)+\right. \\
\left.+\frac{\hbar^{2}}{2 m_{\perp}}\left|\partial_{z} \Psi\right|^{2}+\alpha(T)|\Psi|^{2}+\beta|\Psi|^{4}\right\} .
\end{gathered}
$$


The phenomenological coefficients $\alpha$ and $\beta$ appearing in (1) can be derived in general from experiments or from the microscopic Hamiltonian; the masses $m_{\|, \perp}$ are those of the Cooper pairs. minimizing (11) with respect to $\Psi$. state, hence In the LD tight-binding approach one has a $2 \mathrm{D}$ superfluid wavefunction $\Psi_{n}(x, y)$ in the $n$th plane along $z$ and the term with transverse derivative becomes $-J_{1} \sum_{n}\left|\Psi_{n}(r)-\Psi_{n+1}(r)\right|^{2}$, with $J_{1}$ being the nearest-neighbor tunneling coefficient [2]. spacing of the periodic potential along $z$ ). model from the lattice.

The effective Hamiltonian describing a two-component Fermi gas in a layered optical lattice reads $\hat{H}-\mu \hat{N}$, with the Hamiltonian $\hat{H}$ given by

$$
\hat{H}=-\sum_{\langle i j\rangle \sigma}\left(t_{i j} \hat{\phi}_{i \sigma}^{\dagger} \hat{\phi}_{j \sigma}+\text { h.c. }\right)-U \sum_{i} \hat{\phi}_{i \uparrow}^{\dagger} \hat{\phi}_{i \downarrow}^{\dagger} \hat{\phi}_{i \downarrow} \hat{\phi}_{i \uparrow},
$$

where $\hat{N}=\sum_{i \sigma} \hat{\phi}_{i \sigma}^{\dagger} \hat{\phi}_{i \sigma}$ is the number operator, $\mu$ is the chemical potential, $i, j$ are site in dices, $\sigma=\uparrow, \downarrow$ is the spin index and $\hat{\phi}_{i \sigma}^{\dagger}$ is the fermionic operator (the sum is on distinct pairs of nearest-neighbors). Furthermore $t_{i j}$ is the hopping rate between the sites $i$ and $j$, taken to be equal to $t_{\|}$in the $x y$ planes and $t_{\perp}$ along the $z$ direction. The isotropic 3D case corresponds to $t_{\|}=t_{\perp}$, while the isotropic $2 \mathrm{D}$ case to $t_{\perp}=0$ (isolated pancakes). The singleparticle energies of Hamiltonian (2) for $U=0$ are $\epsilon_{\vec{k}}^{(0)}=-2 t_{\|}\left(\cos k_{x} d+\cos k_{y} d\right)-2 t_{\perp} \cos k_{z} d$ with total bandwidth

$$
D=8 t_{\|}+4 t_{\perp} .
$$

The interaction is assumed attractive, $U>0$, and the total number of fermions per site is denoted by $n$. Estimates of the value of $U$ for which the BCS-BEC crossover occurs gives (in the isotropic case $\left.t_{\|}=t_{\perp}\right) U \approx 0.7 D$ in $3 \mathrm{D}$ and $U \approx 0.2 D$ in $2 \mathrm{D}[22$ 26].

The physical system modeled by (2) has two optical lattices in the $x, y$ directions (determining the tunneling rate $t_{\|}$between the sites in the $x y$ planes) and a different lattice in the $z$ direction, with interlayer coupling $t_{\perp}$ between neighbouring planes. In our subsequent determination of the coefficients of the anisotropic GL and LD models the crucial ingredient is to have the optical lattice along $z$ and the mechanism of layering in the form of tunneling between adjacent layers: if the optical lattices in the $x, y$ directions are absent (or small), then the anisotropic GL (11) would be again retrieved (with $m_{\|}=2 m$, where $m$ is the mass of the fermionic atoms). Similarly, in writing (2) we assumed in each minimum of the lattice there is a total number of fermions $n \leq 2$ (i.e., only the lowest band of the periodic potential is occupied): otherwise, we would have a multi-band Hubbard model, but again a free energy of the form (11) would be retrieved. 


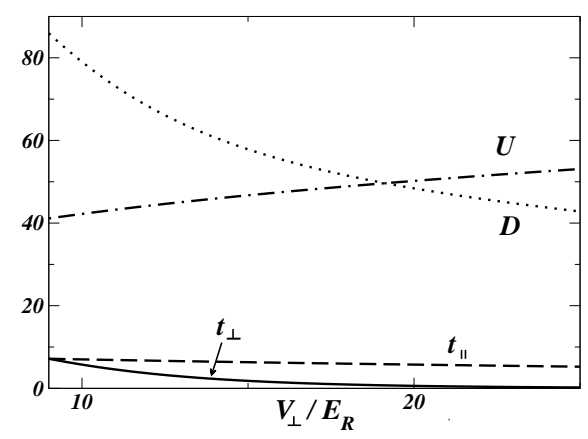

FIG. 1. Values in nanokelvins of the parameters $t_{\perp}$ (solid line), $t_{\|}$(dashed) and $U$ (dot-dashed) as a function of $V_{\perp} / E_{R}$; the total bandwidth $D=8 t_{\perp}+4 t_{\|}$(dotted) is reported as well. Estimates are for ${ }^{6} \mathrm{Li}$ atoms (the parameter $U$ is computed for $|a|=300 a_{0}$ ). The parameter $V_{\|}$is fixed to be $V_{\|}=9 E_{R}$.

\section{ESTIMATES OF PARAMETERS IN REALISTIC POTENTIALS}

The hopping rates $t_{\perp}, t_{\|}$and the interaction coefficient $U$ are as usual expressed as integrals of Wannier functions [9]. We present here estimates for a realistic setup considering an optical lattice potential $V_{\text {opt }}(\vec{r})=V_{\perp}\left[\cos ^{2}(k x)+\cos ^{2}(k y)\right]+V_{\|} \cos ^{2}(k z)$ : the parameters $V_{\perp}, V_{\|}$can be controlled with the power of the laser beams in the $\perp=x, y$ and $\|=z$ directions: the minima of different wells are separated by an energy barrier $V_{\perp}\left(V_{\|}\right)$along the $x, y(z)$ directions. The spacing of the lattice, supposed for simplicity equal in the three directions, is $d=\lambda / 2$ where $\lambda=2 \pi / k$; energies are usually defined in units of the recoil energy $E_{R}=\hbar^{2} k^{2} / 2 m$ [9]. Deep lattices are characterized by having the chemical potential (much) smaller than $V_{\perp}, V_{\|}$. Typical values for the parameters are $\lambda \sim 1 \mu m$ and $V_{\perp}, V_{\|} \sim 1-10 k H z$; for larger values of $\lambda$ (say $\lambda \sim 10 \mu m$ as in [13]) values larger than $500 \mathrm{~Hz}$ are enough to have tunneling dynamics within the first band. For ${ }^{6} \mathrm{Li}$ atoms (using a scattering length $|a|=300 a_{0}$ ), for $V_{\|}=5 E_{R}$ and $V_{\perp}=3.6 V_{\|}$one gets $U / D \approx 0.6$, $t_{\perp} / t_{\|} \approx 0.15$ and $D / k_{B} \approx 60 n K$. For $V_{\|}=9 E_{R}$ and $V_{\perp}=1.25 V_{\|}$one gets $U / D \approx 0.6$, $t_{\perp} / t_{\|} \approx 0.6, D / k_{B} \approx 60 n K$. In Fig:1 we plot the coefficients $t_{\perp}, t_{\|}, U$ vs. $V_{\perp}$ for a set of realistic values of the optical lattice parameters. 


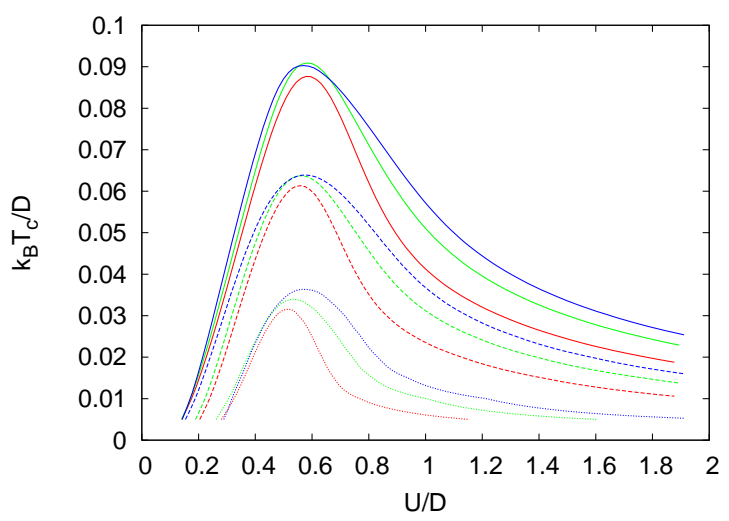

FIG. 2. Critical temperature $k_{B} T_{c}$ vs. $U$ (both scaled in units of $D=8 t_{\|}+4 t_{\perp}$ ) at various particle densities - $n=0.9$ (solid lines), $n=0.5$ (dashed lines), $n=0.1$ (dotted lines) - and anisotropies $t_{\perp} / t_{\|}=1,0.3,0.1$ (in each set from top to bottom).

\section{CRITICAL TEMPERATURE AND CHEMICAL POTENTIAL}

Since the coefficients of (1) depend on the critical temperature $T_{c}$ and the chemical potential $\mu$ - which in turn depend on the parameter of the microscopic Hamiltonian (2) we preliminary study $T_{c}$ and $\mu$ as a function of the interaction $U$, the layering $t_{\perp} / t_{\|}$and the density $n$.

To derive the coefficients of the anisotropic GL we proceed in a path-integral setting [3941]: the fermionic atoms are described by Grassmann fields. First, the Hubbard-Stratonovich transformation is performed; the fermionic degrees of freedom are then integrated out obtaining the bosonic effective action: performing the saddle approximation leads to the meanfield equations. At finite temperature, far from the deep BCS regime (which is obtained for $U \ll D)$, one has to consider the effects of higher order fluctuations [40] and solve two coupled equations one for the gap, the other enforcing the conservation of the number of particles. The first equation is the saddle point equation for the order parameter $\Delta_{0}$ :

$$
\frac{1}{U}=\sum_{\vec{k} \in B Z} \frac{\tanh \left(\beta E_{\vec{k}} / 2\right)}{2 E_{\vec{k}}},
$$

where $E_{\vec{k}}=\sqrt{\epsilon_{\vec{k}}^{2}+\Delta_{0}^{2}}$ and $\epsilon_{\vec{k}}=\epsilon_{\vec{k}}^{(0)}-\mu$. The second equation is the number equation

$$
n=-\frac{\partial}{\partial \mu} F
$$

where $F$ is the free energy, which at the critical temperature (defined by $\Delta_{0} \equiv 0$ ) is given by $F / k_{B} T_{c}=-\sum_{\vec{k} \in B Z} \ln \left(1+e^{-\beta \epsilon_{\vec{k}}}\right)+\sum_{q} \ln \left\{\frac{1}{U}+\chi(q)\right\}$ where $\chi(q)=k_{B} T_{c} \sum_{k} G_{k}^{+} G_{k+q}^{-}$is the 
pair polarization function with $G_{k}^{ \pm}=\left(i \omega \pm \epsilon_{\vec{k}}\right) /\left(\omega^{2}+\epsilon_{\vec{k}}^{2}\right)$ (we used the notation $k=(i \omega ; \vec{k})$ where the wavevectors $\vec{k}$ belong to the Brillouin zone (BZ) and the $\omega_{\ell}$ are the Matsubara frequencies).

To determine the critical temperature one has to solve Eqs. (41)-(5) with $\Delta_{0}=0$ : these equations involves integrals in 6 variables, evaluated first finding the poles of the integrand and analytically integrating around them and then integrating numerically [42]. In this way one obtains $T_{c}$ and $\mu$ as a function of the tunneling rates $t_{\|}$and $t_{\perp}$, the particle density $n$ and the interaction $U$. As an example, for ${ }^{6} \mathrm{Li}$ atoms and for $V_{\|}=9 E_{R}$ and $V_{\perp}=1.25 V_{\|}$ one has $T_{c} \approx 5 n K\left(T_{c} / T_{F} \approx 0.3\right)$. The results for the critical temperature $T_{c}$ for various values of filling $n$ and of the ratio $t_{\perp} / t_{\|}$are plotted in Fig, 2 ; we plot both $T_{c}$ and $U$ in units of $D$, showing that in these units the effect of the anisotropy is relatively small (the critical temperature in the interval $0.5<t_{\perp} / t_{\|}<1$ is very similar to the $T_{c}$ of the isotropic case).

\section{COEFFICIENTS OF ANISOTROPIC GL AND LD MODELS}

The coefficients of the GL free energy (11) are found to be

$$
\begin{aligned}
\frac{\hbar^{2}}{2 M_{a}}= & {\left[\sum_{\vec{k}}\left\{\beta_{c}^{2} X Y+\frac{\beta_{c} Y}{\epsilon_{\vec{k}}}-\frac{2 X}{\epsilon_{\vec{k}}^{2}}\right\} \frac{\left(\partial_{q_{a}} \epsilon_{\vec{k}+\vec{q}}\right)^{2}}{8 \epsilon_{\vec{k}}}+\right.} \\
& \left.+\sum_{\vec{k}}\left\{\frac{X}{4 \epsilon_{\vec{k}}^{2}}-\frac{\beta_{c} Y}{8 \epsilon_{\vec{k}}}\right\} \partial_{q_{a}}^{2} \epsilon_{\vec{k}+\vec{q}}\right]_{\vec{q}=0}, \\
\alpha= & \frac{1}{U}-\sum_{\vec{k}} \frac{X}{2 \epsilon_{\vec{k}}}, \quad \beta=\sum_{\vec{k}}\left\{\frac{X}{4 \epsilon_{\vec{k}}^{3}}-\frac{\beta_{c} Y}{8 \epsilon_{\vec{k}}^{2}}\right\}:
\end{aligned}
$$

in (6) $a=x, y, z$ denotes the direction and the mass parameters $M_{a}$ are related to the masses in the GL energy (11) by $M_{a}=m_{a} U^{2}$ (with $m_{x}=m_{y} \equiv m_{\|}, m_{z} \equiv m_{\perp}$ ). Furthermore it is $X=\tanh \left(\beta_{c} \epsilon_{\vec{k}} / 2\right)$ and $Y=\cosh ^{-2}\left(\beta_{c} \epsilon_{\vec{k}} / 2\right)$ with $\beta_{c}=1 / k_{B} T_{c}$. Eqs. ([6)-(7) are obtained expanding the bosonic action up to the fourth order and evaluating it at the minimum.

The coefficients of (1) depend on the chemical potential $\mu$ and the critical temperature $T_{c}$, which are in turn derived minimizing the free energy evaluated by integrating the gaussian (i.e., at one-loop level) fluctuations above the saddle point of the path integral expression for the partition function. Once that $\mu$ and $T_{c}$ are determined, they are inserted in the coefficients of the GL (11). To account for thermal fluctuations one should keep the GL form 


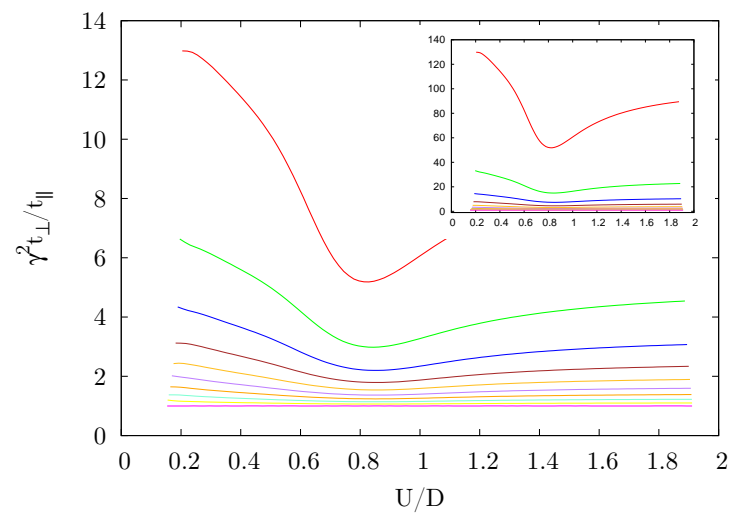

FIG. 3. Effective anisotropy $\gamma_{e f f}^{2}=m_{\perp} t_{\perp} / m_{\|} t_{\|}$as a function of $U / D$ for different anisotropies (at $n=0.5)$ : from top to bottom $t_{\perp} / t_{\|}=0.1,0.2,0.3,0.4,0.5,0.6,0.7,0.8,0.9,1$. Inset: Anisotropy parameter $\gamma^{2}=m_{\perp} / m_{\|}$vs. $U / D$ for the same values of anisotropies $t_{\perp} / t_{\|}$and $n$.

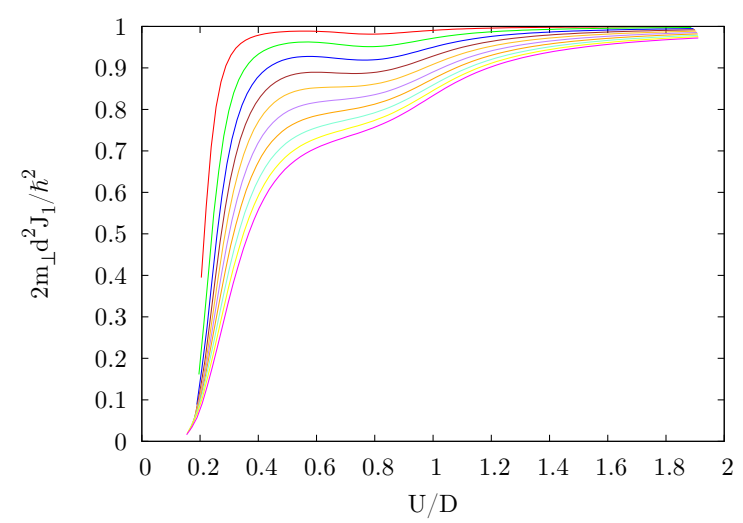

FIG. 4. Plot of the quantity $2 m_{\perp} d^{2} J_{1} / \hbar^{2}$ vs. $U / D$ at $n=0.5$ (with $d$ lattice spacing) for the same values of $t_{\perp} / t_{\|}$of Fig 3

of the effective action and compute observables using the path-integral instead of minimizing the action [43]. Thermal fluctuations play an important role around the unitary limit and in the strongly anisotropic limit, where the behavior of the system is essentially governed by $2 \mathrm{D}$ fluctuations. In particular we expect that our computation is not correct in the pure 2D case, $t_{\|}=0$, and that the range of temperature close to $T_{c}$ at which Eqs. (6)-(7) are valid becomes smaller for $t_{\|} \rightarrow 0$. The validity (and methods of solutions) of the GL equations for $2 \mathrm{D}$ and layered superconductors, also in presence of magnetic fields, has been discussed 444 47]: for ultracold fermions, the comparison with experiments in quasi-2D setups will possibly shed light on the quantitative validity of the GL (1).

It is also possible to derive the LD tunneling coefficients: in the LD model, the $x y$ layers are weakly coupled along the $z$ direction through Josephson terms. Usually, only Joseph- 
son couplings between adjacent planes are considered. Here we consider the more general possibility of longer range tunneling: this amounts to consider a Josephson contribution $-\sum_{n, m} J_{n, m}\left|\psi_{n}-\psi_{m}\right|^{2}$ to the energy, where $n, m$ are denoting the different planes. We find

$$
J_{n, m}=-\frac{U^{2} d}{2 \pi} \int d q_{z} e^{-i(n-m) q_{z} d} \chi\left(i \omega=0 ; 0,0, q_{z}\right)
$$

where $\chi(q)=\chi(i \omega ; \vec{q})$ is the pair polarization function evaluated at the critical point and reported after Eq. (5). In presence of lattices in the $x, y$ directions, one can similarly define Josephson couplings $J_{n, m}^{\|}$. It is possible to determine a useful relation between the coefficients $J_{n, m}$ and the mass $m_{\perp}$ of the anisotropic GL model: one finds

$$
\frac{\hbar^{2}}{2 m_{\perp} d^{2}}=\sum_{p=1}^{\infty} p^{2} J_{p}
$$

where we used the notation $J_{p} \equiv J_{n, n+p}$ (we are assuming translational invariance along $z$ ): then $J_{1}=J_{n, n+1}$.

\section{RESULTS}

Using the solution for $T_{c}$ and $\mu$ of Eqs. (44)-(5) we can use Eq. (6) to evaluate the masses $m_{\|}, m_{\perp}$ and the mass anisotropy parameter $\gamma$ usually defined [2] as

$$
\gamma^{2}=\frac{m_{\perp}}{m_{\|}}
$$

The results for the anisotropy parameter $\gamma$ of a layered ultracold Fermi gas are shown in the inset of Fig.3; $\gamma$ significantly decreases close to the unitary limit due to the effect of the increase of $T_{c}$ in the crossover region. This result is consistent with the qualitative expectation that the effect of external anisotropies is softened in the unitary limit. We investigated the behaviour of $\gamma$ vs. filling, finding that the filling modifies $\gamma$ on the BCS side, while $\gamma$ on the BEC side remains virtually identical. To understand if and how much the collective behavior enhances or not the (external) anisotropy artificially tuned by imposing a layering through a value of $t_{\|} / t_{\perp}$ smaller than one, we defined an effective anisotropy defined as

$$
\gamma_{\text {eff }}^{2}=\gamma^{2} \frac{t_{\perp}}{t_{\|}}=\frac{m_{\perp}}{m_{\|}} \frac{t_{\perp}}{t_{\|}} .
$$

The reason behind the definition (111) is that one expects that the effective mass along the direction $a$ is $\propto 1 / t_{a}$. Then a value of $\gamma_{e f f}$ larger than one signals an effective enhancement 
of the anisotropy (with respect to the "external" anisotropy $t_{\|} / t_{\perp}$ ). The results are drawn in Fig 3 it is seen that in the BCS side the enhancement is more pronounced with respect to the BEC side. Again, the enhancement is reduced in the unitary limit. We also checked that in the BEC limit the quantity $\gamma^{2} \cdot\left(t_{\perp} / t_{\|}\right)^{2}$ approaches 1 for different fillings: a detailed study of $\gamma$ and $\gamma_{e f f}$, as well as of the coefficients $\alpha$ and $\beta$, as a function of the layering across the crossover will be presented elsewhere.

Finally, we numerically evaluated the LD coefficients (8) and compared them to the GL masses in Eq. (6) . We found that in the BEC side the nearest-neighbor Josephson coupling $J_{1}$ is enough to give the correct value $m_{\perp}$ : the numerical estimate of both $m_{\perp}$ and the $J_{p}$ 's show that in the BEC side the identity (91) is very well saturated only by $J_{1}$, i.e. only the $p=1$ term gives an important contribution: therefore in the BEC side one has $J_{1} \approx \hbar^{2} / 2 m_{\perp} d^{2}$.

However, in the BCS side and at the unitary limit our results show that the higher order $J_{p}$ 's (with $p \geq 2$ ) are not negligible, as shown in Fig 4 where we plot the quantity $2 m_{\perp} d^{2} J_{1} / \hbar^{2}$ vs. $U$ : according (9), when this quantity is close to 1 , using only $J_{1}$ is enough to adequately describe the superfluid. A careful numerical investigation of the interlayer couplings $J_{p}$ shows at the unitary limit $J_{2}$ is roughly enough to saturate identity (9), also in the isotropic limit. As example, for $U / D=0.7$, i.e. approximately where there is the unitary limit, one has for $n=0.5$ and $t_{\perp} / t_{\|}=0.6$ that $J_{2} / J_{1} \approx 0.05$; in this case, the identity (9) is satisfied up to $70 \%$ with only $J_{1}$ and up to $95 \%$ with $J_{1}$ and $J_{2}$. This not the case in the BCS side, where other $J_{p}$ 's are needed: however, the deep BCS regime is currently out of reach of experiments since the corresponding critical temperature $T_{c}$ is very low.

The reason for having non-negligible $J_{2}$ (and eventually higher order $J_{p}$ ) is due to the fact that only in the BEC side the size of the pairs is not larger than than the lattice spacing: far form the BEC side the LD model with only nearest-neighbor Josephson couplings is not adequate to describe the superfluid (even though one could think to determine an optimized $J_{1}$ also far from the BEC side and have an effective LD model with only nearest-neighbour tunneling). Fig 4 also shows a non-monotonous behavior around the unitary limit and that the validity region of the LD model with only Josephson couplings $J_{1}$ along $z$ is seen to increase when the layering becomes stronger (i.e., $t_{\perp} / t_{\|}$becomes smaller). 


\section{ANISOTROPY PARAMETER AND EFFECTIVE MASSES}

The anisotropy parameter $\gamma$ plays a crucial role in the electrodynamics of layered superconductors since a formula giving the value of a quantity for a general anisotropy parameter $\gamma$ given its value for $\gamma=1$ holds [48]: the case of the dependence of the upper critical magnetic field upon $\gamma$ has been discussed [49]. Values of $\gamma$ for several superconducting compounds have been determined from experimental data [2].

In layered superconductors, the effects and the penetration of a magnetic field have been studied [2, 50]. They can be incorporated via the minimal substitution $\partial_{a} \rightarrow \partial_{a}-i \frac{e}{c} A_{a}$. $F_{H}=(\vec{B}-\vec{H})^{2} / 8 \pi$. and the response of a layered superconductor to an electromagnetic field have been an established field of research for decades [2, 48, 50]. For ultracold atoms one can simulate a fictitious magnetic field realized by by rotating the trapping potential [52] together with the lattice [53, 54] or by using spatially dependent optical couplings between internal states of the atoms giving raise to a synthetic field [51]. By using a Fermi mixture in optical lattices subjected to a synthetic magnetic field it is possible then to reproduce the phenomenology of the electrodynamics of layered superconductors in the context of ultracold atoms.

In layered Fermi superfluids, the value of $\gamma$ affects the critical value of a synthetic magnetic field (the atomic counterpart of a magnetic field on a layered superconductor [2]). Supposing to have an effective magnetic field $B$ around the axis $a$, at a certain critical value $B_{c 2 ; a}$ the density profile of the cloud will show that the vortices have melt: this could be observed by the disappearance of the vortex cores from the images of the cloud [52]. These critical rotations obey the relation $B_{c 2 \|} / B_{c 2 \perp}=\gamma$.

We finally observe that in experiments with ultracold atomic clouds the effective masses, and then the mass ratio (10), could be experimentally estimated from the measurement of the frequency of the collective excitations. E.g., suddenly moving an external parabolic trap along the $a$ direction, dipole oscillations are induced: the normal (i.e., non-superfluid) part of the cloud would remain on a side of the harmonic potential and eventually roll down up to the center (this since there is an optical lattice in the $a$-direction). However, the superfluid part would oscillate around the center. From the oscillations one can measure the effective masses $m_{a}$ : indeed one has that the frequency without the lattice is $\omega_{a}$ and with the lattice is $\omega_{a} \sqrt{m / m_{a}}$ (this experiment has been already realized with ultracold bosons 
at finite temperature: see e.g. Fig.2(b) of [12]).

\section{CONCLUSIONS}

In this paper we studied effective models for equilibrium properties of the superfluid phase of an ultracold layered Fermi gas in the presence of an optical lattice near the critical temperature. This system is described by an anisotropic Ginzburg-Landau theory (that is also used for other systems such as layered superconductors). We derived and studied the coefficients of the Ginzburg-Landau equation: the anisotropy was shown to be enhanced by the superfluid, and the anisotropy parameter $\gamma$ decreases near the crossover region. The dependence on filling was found to be important on the BCS side of the crossover and negligible in the BEC side. Possible experimental measurements of the effective masses and the anisotropic parameter have been as well discussed. We also derived the LawrenceDoniach model for the layered ultracold Fermi gas and we discussed a relation between the interlayer Josephson couplings in the Lawrence-Doniach model and the masses in the Ginzburg-Landau energy. We found that using only couplings between adjacent planes is correct in the BEC side: at the unitary limit one can limit oneself to the use of only $J_{1}$ and $J_{2}$, while in the BCS side contributions from longer range interlayer couplings appear.

The obtained results link the underlying microscopic description of a layered ultracold Fermi gas to the macroscopic properties of the superfluid and show that tuning the experimentally controllable parameters such as interaction strength, filling and lattice strength it is possible to emulate the phenomenological properties of layered superconductors.

\section{ACKNOWLEDGMENTS}

Discussions with K. Schmidt and G. Roati are gratefully acknowledged.

[1] Lawrence W.E. S. Doniach, in Proc. 12th Int. Conf. Low Temp. Phys., ed. E. Kanda, p. 361 (Tokyo, Keikagu) 1971.

[2] Tinkham M., Introduction to superconductivity (New York, McGraw-Hill) 1996.

[3] Gamble F.R. et al., Science, 168 (1970) 568. 
[4] Ruggiero S.T. et al., Phys. Rev. Lett., 45 (1980) 1299.

[5] Hannay N.B. et al., Phys. Rev. Lett., 14 (1965) 225.

[6] Klemm R.A. et al., Phys. Rev. B, 12 (1975) 877.

[7] Bednorz J.G. Müller K.A., Z. Phys. B, 64 (1986) 189.

[8] Leggett A.J., Quantum liquids: Bose condensation and Cooper pairing in condensed-matter systems, Chap. 7 (Oxford, Oxford University Press) 2006.

[9] Morsch O. Oberthaler M.K., Rev. Mod. Phys., 78 (2006) 179.

[10] Bloch I. et al., Rev. Mod. Phys., 80 (2008) 885.

[11] Greiner M. et al., Nature, 415 (2002) 39.

[12] Cataliotti F.S. et al., Science, 293 (2001) 843.

[13] Albiez et al., Phys. Rev. Lett., 95 (2005) 010402.

[14] Modugno G. et al., Phys. Rev. A, 68 (2003) 011601.

[15] Chin J.K. et al., Nature, 443 (2006) 961.

[16] Stöferle T. et al., Phys. Rev. Lett., 96 (2006) 030401.

[17] Schneider U. et al., Science, 322 (2008) 1520.

[18] Jordens R. et al., Nature, 455 (2008) 2004.

[19] Sommer A.T. et al., Phys. Rev. Lett., 108 (2012) 045302.

[20] Titvinidze I. et al., New J. Phys., 12 (2010) 065030.

[21] Hofstetter W. et al., Phys. Rev. Lett., 89 (2002) 220407.

[22] Singer J.M. et al., Phys. Rev. B, 54 (1996) 1286.

[23] Sewer A., Zotos X., Beck H., Phys. Rev. B, 66 (2002) 140504.

[24] Toschi A. et al., New J. Phys., 7 (2005) 7.

[25] Burovski E. et al., New J. Phys., 8 (2006) 153.

[26] Chien C.C., Chen Q.J., Levin K., Phys. Rev. A, 78 (2008) 043612.

[27] Orso G. Shlyapnikov G.V., Phys. Rev. Lett., 95 (2005) 260402.

[28] Iskin M. Sá de Melo C.A.R., Phys. Rev. Lett., 103 (2009) 165301.

[29] Fialko O. et al., Phys. Lett. A, 374 (2010) 3869.

[30] Watanabe G. et al., Phys. Rev. A, 83 (2011) 033621.

[31] Günter K. et al., Phys. Rev. Lett., 95 (2005) 230401.

[32] Du X. et al., Phys. Rev. Lett., 102 (2009) 250402.

[33] Martiyanov K. et al., Phys. Rev. Lett., 105 (2010) 030404. 
[34] Fröhlich B. et al., Phys. Rev. Lett., 106 (2011) 105301.

[35] Dyke P. et al., Phys. Rev. Lett., 106 (2011) 105304.

[36] Feld M. et al., Nature, 480 (2011) 75.

[37] Giorgini S. et al., Rev. Mod. Phys., 80 (2008) 1215.

[38] Ginzburg V.L. Landau L.D., Zh. Eksp. Teor. Fiz., 20 (1950) 1064.

[39] Sá de Melo C.A.R. et al., Phys. Rev. Lett., 71 (1993) 3202.

[40] Randeria M., in Bose-Einstein condensation, eds. A. Griffin et al. (Cambridge, Cambridge University Press) 1995.

[41] The BCS-BEC Crossover and the Unitary Fermi Gas, ed. W. Zwerger (Heidelberg, Springer) 2012.

[42] Iazzi M., Ph.D. Thesis ((Trieste, SISSA)) 2012.

[43] Larkin A.I. Varlamov A.A., Theory of fluctuations in superconductors (Oxford, Clarendon) 2005.

[44] Ullah S. Dorsey A.T., Phys. Rev. B, 44 (1991) 262.

[45] Tes̆anović Z. Andreev A.V., Phys. Rev. B, 49 (1994) 4064.

[46] Benfatto L. et al., Eur. Phys. J. B, 13 (2000) 609.

[47] Murray J.M. and Tešanović Z., Phys. Rev. Lett., 105 (2010) 037006.

[48] Blatter G. et al., Phys. Rev. Lett., 68 (1992) 875.

[49] Mineev V.P., Phys. Rev. B, 65 (2001) 012508.

[50] Rosenstein B. Li D., Rev. Mod. Phys., 82 (2010) 109.

[51] Lin Y.J. et al., Nature, 462 (2009) 628.

[52] Fetter A.L., Rev. Mod. Phys., 81 (2009) 647.

[53] S. Tung et al. Phys. Rev. Lett., 97 (2006) 240402.

[54] Williams R.A. et al., Phys. Rev. Lett., 104 (2010) 050404., 